\title{
Experience and coping strategies of women victims of domestic violence and their professional caregivers: a qualitative study
}

\author{
Elaheh Asadi-Bidmeshki ${ }^{1}$, Jamileh Mohtashami², Meimanat Hosseini ${ }^{3}$, Seyyed Mehdi Saberi ${ }^{4}$, \\ Fiona Nolan 5 \\ 1PhD Student in Nursing, Student Research Committee, School of Nursing and Midwifery, Shahid Beheshti University \\ of Medical Sciences, Tehran, Iran \\ ${ }^{2}$ Department of Psychiatric Nursing, School of Nursing and Midwifery, Shahid Beheshti University of Medical Sciences, \\ Tehran, Iran \\ ${ }^{3}$ Department of Community Health Nursing, School of Nursing and Midwifery, Shahid Beheshti University of Medical \\ Sciences, Tehran, Iran \\ ${ }^{4}$ Department of Psychiatry, Legal Medicine Research Center, Legal Medicine Organization, Tehran, Iran \\ ${ }^{5}$ Florence Nightingale Foundation Clinical, Nursing School of Health and Social Care, University of Essex, UK
}

Neuropsychiatria i Neuropsychologia 2021; 16, 1-2: 92-100

\author{
Address for correspondence: \\ Dr Jamileh Mohtashami, Assoc. Prof. \\ Department of Psychiatric Nursing \\ School of Nursing and Midwifery \\ Shahid Beheshti University of Medical Science \\ Tehran, Iran \\ e-mail: j_mohtashami@sbmu.ac.ir
}

\section{Abstract}

Introduction: Domestic violence is associated with many negative and potentially long-lasting consequences to women's mental, physical, and sexual health. This study aimed to identify strategies which had been used by women who had been victims of domestic violence.

Material and methods: This qualitative content analysis was conducted using semi-structured interviews from 1 February 2019 to 1 August 2019 in Tehran. Participants were female victims of domestic violence and their health care providers recruited from Forensic Medicine centers, clinics, hospitals and Welfare Organizations of Tehran.

Results: From these twenty-six interviews we extracted five main themes: emotional introjection, pragmatic actions against violence, sheltering under the supportive umbrella, seeking refuge to avoid violence and remaining in the violent environment.

Conclusions: The experiences of our sample of women and of professionals involved in their care indicated that domestic violence was tolerated by some for financial, cultural and social reasons, and this had severe psychological impacts on the victims. Others from our sample were enabled to act to change their abusive situations but with support from their family or from wider society. The information obtained can potentially be used to inform the design of services and interventions for women who are victims of domestic violence.

Key words: female, sexual health, domestic violence.

\section{Introduction}

Violence is a threat to the lives, health, and happiness of all people. Everyone has the right to live in peace in their homes, but sometimes women's rights to do so are violated due to violence (Khatri and Pandey 2013). Prevalence of domestic violence among women victims is $36.2 \%$.

A World Health Organization (WHO) report highlighted that domestic violence against women was the leading cause of death and injury to women worldwide and defined it as a factor negatively affecting individuals' health status (Gómez-Fernández et al. 2017). The United Nations (UN) describes violence against women as a "gender-based violence and a leading cause of physical, sexual, or psychological harm or suffering to women". On the other hand, the threat of violence, coercion or arbitrary deprivation of liberty, both in public and private life, has also been defined as violence against women (WHO 2014).

Domestic violence has been defined as taking one of four types: physical violence, such as beating, psychological violence including humili- 
ation, restriction of liberty, profanity, disregard for the feelings and emotions, sexual violence, including sexual coercion through intimidation and threats in an unwanted time and place, performing painful or harmful acts and economic violence, including depriving women of their basic needs, causing deprivation of possessions, forcing women into an unwanted job, preventing women from working or threatening such acts (Adjah and Agbemafle 2016; Shuib et al. 2013; Uzun and Uzunboylu 2015).

In 2014 the WHO reported that domestic violence against women occurred in $30 \%$ (Abramsky et al. 2016). A study by Taherkhani et al. conducted in 2010 in Tehran reported that the prevalence of psychological, physical, and sexual abuse within their sample of 811 women was $87 \%, 25 \%$, and $39 \%$, respectively (Vakily et al. 2017). Khangholi et al. (2019) reported that almost one-third of 150 Iranian women interviewed in 2019 were subject to their sexual partner's violence during their lifetime and domestic violence occurred among $21.45 \%$ of couples.

Violence is seen as a serious barrier to women's advancement (Shuib et al. 2013). Two studies have found that women subjected to violence are 4-6 times more likely to be depressed than those who are not (Al-Natour et al. 2016). Furthermore, the risk of mental illnesses such as eating disorders, anxiety, post-traumatic stress syndrome, and suicide is higher in these women (Adjah and Agbemafle 2016; Bradbury-Jones et al. 2017). However, cultural and legal norms strongly influence the ability of women to leave these environments. Violence against women is perceived as justified in some cultures, such as Iran, for reasons such as failure to perform housework, being unfaithful to their husband or because their clothing or behavior displeases their husband. In many cases, women do not ask for help due to shame, fear, denial, lack of social and legal protection and self-reproach (Garrusi et al. 2008; Sadeghi-Fasaee 2010).

Various coping strategies are adapted by different individuals confronted with negative affective states and associated life problems due to domestic violence (Itimi et al. 2014). Previous authors have identified that victims of violence use specific methods to cope with their situation with mixed success in reducing the violent behavior or the resulting psychological impact (Carvalho et al. 2019; Itimi et al. 2014; Khangholi et al. 2019). Coping has been described as a dynamic process that involves constantly changing cognitive and behavioral efforts, and is managed by internal and external needs that are beyond one's resources (Taherkhani et al. 2016). Given the influence of cultural and contextual factors, examining coping strategies in women subjected to domestic violence has the potential to extend the current knowledge on the way that abused women cope with domestic violence. Therefore, the reason for the prevalence of violence in Iran (27-83\%) can be seen as directly linked to the patriarchy culture and women's control in some cities (Amini et al. 2014) and its harmful effects on the quality of life of women and their children (Noorisanchooli et al. 2018). This study aimed to build on existing evidence for effective strategies to counter domestic violence through exploring the experience of victims and the views of those who have been intimately involved in their care and treatment.

\section{Material and methods}

This study is part of a multi-stage study which uses qualitative study and a systematic literature review to develop a care plan for women under intimate partner violence. We used qualitative interviews to explore the personal experience of participants. This method was chosen by the authors as a suitable means by which to understand hidden feelings and meanings in the experiences of human life (Pouraboli et al. 2014).

\section{Study sites}

Participants were recruited from 3 hospitals in different geographical settings throughout Tehran, 4 clinics based in hospitals in Tehran, one of which provided psychological support to victims of violence and the other three only screened and referred, two forensic medicine centers that only conducted forensic examinations and two welfare centers, one for social emergency services and a safe house, and the other for a welfare-affiliated charity.

Our inclusion criteria for service users were: women who were under the care of professionals at one of the study sites, who were identified by a psychiatrist clinical psychologist, nurse, midwife or other professionals as having been victims of domestic violence at the hands of their partner or spouse, were able to speak and read Persian and had capacity to consent to participate.

The inclusion criteria for professionals was having experience in identifying, treating and caring for women who were victims of domestic violence. This included doctors, nurses, midwives, family health experts, and 
psychologists. Participants needed to be employed and involved in caring for the service user participants in Tehran Forensic Medicine Center, Welfare or one of the hospitals or clinics at the time of recruitment.

We used a convenience sampling method and the referrers selected potential participants based on whether they felt they could speak at ease and eloquently about their experiences, thereby providing rich and expansive data sources from which to extract our themes. The data collection instrument consisted of deep and semi-structured interviews through open-ended questions, such as Describe one of your domestic violence experiences or What do you do when you are abused? (asked of women victims of domestic violence) and Describe the experience of caring for a women under domestic violence (asked of professional caregivers). The interview questions were made by research team members and the first interview was considered as pilot.

The women victims of domestic violence in this research were selected based on the introduction of the representative of the head of the center of mentioned centers considering the inclusion criteria. The sampling continued until data saturation. The saturation involved repetition and confirmation of the previously collected data. In other words, repetition of previous information or repetition of themes suggests adequacy of sample size (Polit and Beck 2012).

In this research, participants were first authorized by the representative of the head of the center to refer to the researcher if they wished to participate in the study. After obtaining permission, the contact information was provided to the researcher. Then the interviewer (the first author) invited participants for an interview. The aim of the study was explained and then the interviews were conducted in a quiet and peaceful environment selected by the participant and agreed with the researcher. The duration of interviews ranged from 25 to 45 minutes. All interviews were electronically recorded, and transcribed. Coding of the data was conducted by two researchers (EAB, JM) concurrently with data collection, to facilitate decisions around saturation. The analysis was carried out according to the five stages proposed by Graneheim and Lundman (2004), which are 1 . Recording the interview and transcribing immediately, 2 . Reading the entire text of the interview to achieve a general understanding of its content, 3 . Determining the meaning of units and preliminary codes, 4. Classifying similar preliminary codes in more comprehensive classes and 5. Determining the hidden content in the data.

A conventional content analysis method was used. To interpret the direction and rigor of data, four criteria - validity, reliability, confirmability, and transferability - were used (Lincoln and Guba 1986). To ensure the validity, after analyzing each interview, the text of interviews was referred again to the participants, where the accuracy of the points was confirmed and the necessary modifications were made. In order to interpret the confirmability, the researchers tried not to introduce their presumptions into the data collection and analysis as much as possible. The analysis and coding were discussed within the wider study team comprising five professionals and a range of expertise.

\section{Ethical considerations}

The study was approved by the ethics committee of the Faculty of Nursing and Midwifery of Shahid Beheshti University of Medical Sciences (IR.SBMU.PHNM.1396.1006). In accordance with the approved processes the study team provided information to the forensic medical centers, clinics, hospitals, and the welfare organization of Tehran and after obtaining approval for the study to be conducted there, from the head of the mentioned centers, information about the study was provided verbally by the study researchers in a face-to-face meeting with each potential participant, and written consent was obtained prior to interviews. Participants were allocated study numbers and the list linking their names with these numbers was accessible only to the first author. Participants were assured that their data were confidential and that they could withdraw from the study at any point.

\section{Findings}

Fifteen service users and sixteen professionals across eleven study sites were approached to participate. Of these, twelve and fourteen respectively agreed. Personal characteristics are presented in Tables 1 and 2 . Three of the service users refused due to fear of their husbands and two staff did not participate because they were so busy and did not have time. All participants were female in both groups. The reason that professionals were female was because services provided to women in Iran are required to be provided by female professionals only. Data analysis produced 5 main themes and 49 themes across both groups: emotional introjection (due to spouse's violence), pragmatic actions against 
Table 1. Characteristics of service users $(n=12)$

\begin{tabular}{|c|c|}
\hline \multicolumn{2}{|l|}{ Parameter } \\
\hline Mean age (years) & $35.6 \pm 9.5$ \\
\hline \multicolumn{2}{|l|}{ Level of education } \\
\hline Academic education & 4 \\
\hline Diploma & 6 \\
\hline Undergraduate & 2 \\
\hline \multicolumn{2}{|l|}{ Marital status } \\
\hline Married & 7 \\
\hline Divorced & 3 \\
\hline Separated & 2 \\
\hline \multicolumn{2}{|c|}{ Employment outside the home } \\
\hline No & 8 \\
\hline Yes & 4 \\
\hline
\end{tabular}

violence, sheltering under the protective umbrella, seeking refuge for avoiding violence and remaining in the violent environment (Table 3).

\section{Emotional introjection (due to spouse's violence)}

Following the repetition and intensification of violence, some of the feelings and reactions were triggered in the victim and were usually emotional introjection by the individual. Themes of this main theme included wishing to die, feeling hopelessness in the marriage, running out of patience, desperation, vulnerability, violenceinduced mental disorder, loss of maternal feeling, and closeness to God.

In relation to 'running out of patience' Professional 14: When I talk to women referring here, they often say that they are now in the end stages of their married life, are tired, and are getting belp from the governmental organization to help them avoid domestic violence.

Professional 7 said about violence-related mental disorder:

In fact, one cannot say that the injuries to the female clients are definitely only physical because they have been suffering from these injuries for many years. Many years have passed, and the violence has made its mental impacts; they now refer to us suffering from a physical event or injury; but their mental dimension is definitely affected.

\section{Pragmatic actions against violence}

Some participants described taking action to prevent future violence. Sub-themes which we identified here include asking for help, financial independence, protective behaviors, spouse conviction, legal prosecution process, and secur-
Table 2. Characteristics of professionals $(n=14)$

\begin{tabular}{lc} 
Parameter & $39.7 \pm 8.4$ \\
\hline Mean age (years) & $14.5 \pm 8.2$ \\
\hline Mean years of work & 6 \\
\hline Level of education & 3 \\
\hline Bachelor & 3 \\
\hline Master's degree & 2 \\
\hline PhD
\end{tabular}

ing the environment. These are exemplified by Participant 6:

I filed a complaint and came to the Department of Forensic Medicine to frighten him and make him never beat me anymore. I want him to stop beating me.

Participant 11:

When my husband beat me, I would just sit in a corner, cover my head with my hands, and say notbing.

\section{Remaining in the violent environment}

The experiences of participants and professional caregivers in the study indicated that certain personal or social conditions or barriers lead the victims not to disclose violence or tolerate it and that the victim of violence cannot therefore leave the violent environment that include doubt, trying to preserve life/remaining hopeful, misplaced trust in the perpetrator, lack of exhibiting response to violence, individual barriers to divorce/leaving a violent relationship, legal barriers to pursuing a complaint, barriers to expressing and reporting violence (reluctance to disclose violence), and financial barriers to following up care.

Participant 1 said about legal barriers to pursuing a complaint:

After years of harassing me in different ways, I finally went to the sheriff's office, filed a complaint, went to the court, and said to the judge: My husband brings home some women, shows violence to me, harasses me..., but nothing special happened, meaning no punishment or retribution was considered for him because they said you must present evidence, such as evidence of addiction, or admissible evidence suggesting his relationship with other women.

When they are asked about the violence, they first deny it because many of them have respectable families, and fear that their husbands would threaten their families, and cause injury to their families; for this reason, they deny it as much as possible.

\section{Sheltering under the supportive umbrellas}

Experiences of participants and professionals indicated that some care and supportive mea- 
Table 3. Themes

\begin{tabular}{|c|c|}
\hline Main themes (5) & Themes (49) \\
\hline $\begin{array}{l}\text { Emotional intro- } \\
\text { jection (due to } \\
\text { spouse' violence) }\end{array}$ & $\begin{array}{l}\text { - } \text { wishing to die } \\
\text { - frustration of married life } \\
\text { - run out of patience } \\
\text { - desperation } \\
\text { - vulnerability } \\
\text { - violence-induced mental disorder } \\
\text { - loss of maternal feeling } \\
\text { - closeness to God }\end{array}$ \\
\hline $\begin{array}{l}\text { Pragmatic } \\
\text { actions against } \\
\text { violence }\end{array}$ & $\begin{array}{l}\text { - } \text { asking for help } \\
\text { - financial independence } \\
\text { - protective behaviors } \\
\text { - spouse conviction } \\
\text { - legal prosecution process } \\
\text { - securing the environment }\end{array}$ \\
\hline $\begin{array}{l}\text { Sheltering under } \\
\text { supportive um- } \\
\text { brellas }\end{array}$ & $\begin{array}{l}\text { - } \text { attempts for initial assessment } \\
\text { - } \text { paving the way for legal support } \\
\text { - psychiatric care } \\
\text { - confirmation of violence } \\
\text { - drug therapy, } \\
\text { - treatment of violence perpetrators } \\
\text { - counseling, referral } \\
\text { - charitable services } \\
\text { - screening } \\
\text { - providing security } \\
\text { - intersectoral collaboration } \\
\text { - self-care education } \\
\text { - public education } \\
\text { - awareness raising } \\
\text { - disclosure of violence following } \\
\text { effective communications with } \\
\text { - caregivers } \\
\text { - emergency social measures } \\
\text { - empowerment, entrepreneurship/ } \\
\text { - the role of philanthropists } \\
\text { - the process of being admitted to } \\
\text { - safe homes, } \\
\text { - family/relative support } \\
\text { - } \text { financial support }\end{array}$ \\
\hline $\begin{array}{l}\text { Seeking refuge to } \\
\text { avoid violence }\end{array}$ & $\begin{array}{l}\text { - } \text { expectation from the judicial } \\
\text { - } \text { system } \\
\text { - } \text { need to communicate } \\
\text { - } \text { required support }\end{array}$ \\
\hline $\begin{array}{l}\text { Remaining in the } \\
\text { violent environ- } \\
\text { ment }\end{array}$ & $\begin{array}{l}\text { - } \text { staying due to having doubt } \\
\text { - } \text { hopefung to preserve life/remaining } \\
\text { - misplaced trust in the perpetrators } \\
\text { - lack of response to violence } \\
\text { - individual barriers to divorce/le- } \\
\text { aving a violent relationship } \\
\text { - legal barriers to pursuing com- } \\
\text { - plaints } \\
\text { barriers to expressing and } \\
\text { reporting violence (reluctance to } \\
\text { disclose the violence) } \\
\text { - financial barriers to following up } \\
\text { care }\end{array}$ \\
\hline
\end{tabular}

sures, such as screening, played a significant role in identifying, providing appropriate care and guidance, and helping to make effective decisions. Comprehensive care (physical, psychological, social) for women victims of violence, and the treatment of their spouses at the same time, is an important issue and the treatment of perpetrators of violence has often been neglected. The intersectoral collaboration between government organizations and the need to coordinate these organizations, the important role of nongovernmental organizations (NGOs) and private organizations and the role of philanthropists and charities, having a strong support system, education, both individual and public, the emotional and financial support of the family were also an important pillar of support and played an important role in helping women victims of violence to leave the violent environment.

Professional 1 said on initial assessment as follows:

We write down all the bruises in our assessment sheet the very first time a woman enters the ward, we call and tell the doctor if there is something that needs treatment, for example, there is blood in her hair, we ask: what's wrong? She says, for example: My husband pushes me.

Professional 4 said about the treatment of a violence perpetrator:

If their spouse suffers from a psychological problem, we persuade him, if necessary, to come and take drugs under the supervision of the doctor.

\section{Seeking refuge to avoid violence}

Participants shared their expectations of the judiciary and the health care system to approach violence, which in turn determines the care needs of victims. Subthemes included the expectation of the role of a judicial system, the expected health care, the need for communication with others, and the support needed.

Participant 7 described her expectations from the judiciary as follows: I expect to really take care of our women's rights. Look, I've been out of my busband's bouse for 4 or 3 months now. But there is no law that allows me to be divorced.

Table 4 shows how a main theme forms from the meaning units and initial codes.

\section{Discussion}

The experiences of women victims of domestic violence and their professional caregivers that indicated women's strategies for dealing with domestic violence were in two forms: emotional introjection (due to spouse' violence) or prag- 
Table 4. An example extraction of codes, themes, and main themes from meaning units

\begin{tabular}{|c|c|c|c|}
\hline Example of meaning units & Example of codes & Themes & Main themes \\
\hline $\begin{array}{l}\text { Participant 6: I told my husband } \\
\text { I would forgive my marriage } \\
\text { portion but you don't bother me } \\
\text { so much. }\end{array}$ & $\begin{array}{l}\text { Offer to reduce the dowry } \\
\text { to stop harassment }\end{array}$ & $\begin{array}{l}\text { Trying to preserve life/ } \\
\text { remaining hope }\end{array}$ & $\begin{array}{c}\text { Remaining in a violent } \\
\text { environment }\end{array}$ \\
\hline $\begin{array}{l}\text { Professional 4: I had a client } \\
\text { whose husband had told her } \\
\text { to go out of his house because } \\
\text { I couldn't buy a new house for } \\
\text { my second wife so it would be } \\
\text { better for me to divorce you and } \\
\text { bring her in. That woman had } \\
\text { told me that she had no problem } \\
\text { and she can come and live with } \\
\text { us, that is, it shows an absolute } \\
\text { mental collapse of this woman } \\
\text { who is ready not to lose this life } \\
\text { in complete humiliation and } \\
\text { helplessness and for that lady to } \\
\text { enter her home. }\end{array}$ & $\begin{array}{l}\text { Woman agrees to live with } \\
\text { second wife in one place }\end{array}$ & & \\
\hline
\end{tabular}

matic actions against violence. The type and severity of these reactions varied in the women, with some not reacting, remaining in the violent environment, and others trying to avoid the violence and finding refuge with others and raising their expectations for an approach to violence.

While confronting different dimensions of violence and responding to domestic violence, some women had emotional introjection due to spouse's violence, in that some were frustrated with their married life due to the severity of the violence and experienced some degree of psychological disorders caused by the violence. Running out of patience, feeling of despair, and vulnerability were so severe that some women wished to die. Previous studies have referred to violence-related consequences including substance abuse, depression, post-traumatic stress and other anxiety and somatoform disorders (Kalra et al. 2017), low self-esteem, suicidal ideation and suicide (Chepuka et al. 2014), and poor quality of life in women victims of domestic violence. Continued violence and lack of social support contribute to psychological disorders (Beeble et al. 2009). Also, the maternal feeling was hurt in some women victims of domestic violence due to feelings of despair, helplessness, and confusion, and some of them noted that they had been forced to award the custody of their children to welfare organizations or other relatives in spite of their inner desires and were consequently very worried. In some cases, some women found comfort in religion. This echoes the findings from one study in Iran which indicated that women victims of domestic violence had a stronger religious attitude and higher flexibility (Dehghani-Firoozabadi et al. 2017).

Some women, however, resorted to pragmatic actions. Some sought assistance from responsible organizations and institutions, such as a social emergency or forensic medicine. To achieve financial independence and exit the violent environment, some women saved money or found employment. Some adopted protective behaviors such as leaving home, shouting, informing neighbors, protecting their heads and neck. In Pallitto et al.'s study, these behaviors included protecting children from violence, developing safety strategies, and accessing social resources for social support (Pallitto et al. 2016).

Some women tolerated their situation due to hesitation to leave the violent environment and trying to save lives at any cost. In most cases, they trusted the perpetrator. Some reacted passively and remained silent, an issue that has been addressed in other studies. Women have largely adapted to domestic violence and many have suffered in silence (Krishnan et al. 2012). However, the factors that played an important role in making these women tolerate the violent environment included the individual, financial, or social barriers to leaving the violent relationship. Barriers such as maintaining dignity, having children, fear, lack of support in family and society, lack of financial independence, cultural, social, and economic consequences of divorce made a person unable to leave the violent relationship or unwilling to disclose the violence. Other studies have also pointed to the barriers to leaving a violent relationship. Women cannot 
always leave their violent partners with ease of mind (Pallitto et al. 2016). Despite the difficult circumstances and difficulties in marital life, women suffering domestic violence remained in those situations and endured those difficulties to avoid the consequences of divorce (social, psychological, legal, financial) (Noorisanchooli et al. 2018). It seems that despite the possibility of leaving a violent relationship, women are being held captive in a violent situation by the fear of experiencing more violence against themselves and their children, lack of financial resources, lack of family support, and lack of legal and social assistance (Crombie et al. 2017; Golu 2014). Concerns for children and the hope that the spouse will change his behavior are common reasons for staying in a violent relationship (Dicola and Spaar 2016).

In some cases, legal barriers, such as a complicated and long legal process in courts, the time-consuming and ineffective legal process, and absence of sufficient evidence to prove the infidelity or addiction of the spouse forced the person to remain in a violent environment. Most governments have considered domestic violence against women as a minor social misunderstanding, a private matter, and have not recognized it as a crime (Semahegn et al. 2017).

Some women sought supportive umbrellas and wanted to protect themselves or to avoid violence by receiving supports from family or responsible organizations. Support services provided by professional caregivers included initial assessment and approval of violence and drug therapy. McFarlane et al. (2006) stated that when nurses assess women for spousal abuse and the abused women decide to disclose violence, the violence is confirmed, her experience validated, and the search for assistance becomes legitimate. To lay the groundwork for legal support was another step taken by professional caregivers and includes recording of symptoms, the preparation of evidence, and attaching them to the patient's case to prepare the ground for legal pursuit. As noted in Kalra's study, care providers can play an important role in collecting and documenting the evidence needed to identify and take legal action against perpetrators of violence (Kalra et al. 2017). Another support aspect provided by care providers was mental health care, which included counseling and referral to a psychiatrist or psychologist, drug therapy and hospitalization if necessary. In this regard, resistance of abused women, or intimate partner violence (IPV) perpetrators or their family, was a barrier to care, and follow-up of treatment was not possible without the consent of the individual or her family. In a psychological intervention study involving a variety of cognitive-behavioral psychotherapy methods, they led to clinical improvement of mental health outcomes compared to standard supportive care (Ferrari et al. 2018). Treatment of the violence perpetrator was an important measure suggested by caregivers to the perpetrator since women victims of violence reported that their spouse had been suffering from unreasonable suspicion, uncontrolled anger, or drug abuse. However, in most cases, the perpetrator did not accept treatment or refused to continue it. Studies have revealed that combined treatments that simultaneously resolve problems caused by drug abuse and violence have the lowest recurrence rates (Stover et al. 2009). Some support services were provided by organizations such as welfare centers, charities, and NGOs that ensured good support but were insufficient due to lack of financial and human resources, as well as insufficient information on these services. These support services included entrepreneurship/employment, charity and secure home services. In a randomized controlled trial of women victims of violence who resided in shelters in Michigan and participated in a 10-week support program, those who received support were less likely to be victims of violence in the next two years than the control group (McCloskey et al. 2006). Empowerment was provided also, included counseling and training on self-protection, finding a job and life skills training. Harvey et al. (2018) noted that empowering women and promoting gender equality is one of the 17 Sustainable Development Goals (SDGs) set out in the 2030 Agenda for Sustainable Development, adopted by all UN member states in 2015 (Harvey et al. 2018) and reduces the prevalence of IPV and improves women's health status (Bott et al. 2005; García-Moreno et al. 2015; Tiwari et al. 2005). Furthermore, the financial support provided mostly through the welfare organization and sometimes in the form of home loan prepayment to meet the initial needs was not sufficient due to limited resources. The results of a study showed that absence of a sponsor was associated with an increased risk of IPV and repeated cases of violence during pregnancy (Sigalla et al. 2013). In some cases, simply informing IPV victims about available resources and services can be considered as an intervention. Some women participating in the study by Alvarez et al. (2016) stated that if nobody had told them about social services, they would never have been informed and perhaps 
never left the violent relationship. The WHO recommends legal reforms and media campaigns to raise awareness of IPV. School-based education programs on domestic violence have shown a reduction of gender-based violence. It has been shown that early intervention services in at-risk families reduce child maltreatment and may reduce subsequent violent behaviors in life (Dicola and Spaar 2016).

However, women deprived of family support were forced to stay in the violent environment and tolerate violence due to lack of support. Sigalla et al. (2013) said that talking to a family member at least once a month contributed to a reduction in incidence and recurrence of IPV during pregnancy.

Women victims of domestic violence had some expectations for avoiding violence, including expectations from the judiciary, health care, support, and communication. Kalra et al. (2017) also stated that effective responses include listening empathetically, validating victims' feelings, knowing when and how to ask about violence, discussing victims' violence and readiness to change, psychological support, encouraging safe behaviors for victims of intimate partner violence, and identifying and reporting violence with full documentation as well as the referral of victims to existing specialized organizations (Kalra $e t$ al. 2017). Supportive interventions (i.e., information and support to combat violence and access to services needed) can improve women's quality of life, safety measures, social support and access to services, and lead to a reduction in violence, especially among those who disclose violence or seek help from shelters (Jack et al. 2012).

A limitation would be that the study is a small qualitative evaluation, and therefore limited in ability to generalize. Therefore we recommend that larger scale research should be conducted within this group in order to explore the issues raised across a wider population of victims, their families, perpetrators, policy makers and professionals.

\section{Conclusions}

Overall, the experiences of abused women and their professional caregivers indicated that some women, despite the severity of the violence for various reasons (financial, cultural, social), endured the violence and remained in the atmosphere of violence and showed emotional introjection. And others, in seeking refuge to avoid violence and support of the family or community, take action to prevent domestic violence.
It is recommended that relevant organizations and institutions establish and strengthen support resources through inter-sectorial collaboration and coordination and raise public awareness of these resources through proper information to community members, especially abused women.

\section{Acknowledgments}

This study is a part of a doctoral thesis at the School of Nursing and Midwifery, Shahid Beheshti University of Medical Sciences, Tehran. The authors would like to thank the participants who shared their experiences, as well as authorities of the forensic medicine, clinics, hospitals, and the welfare organization who cooperated significantly in this research.

\section{Disclosure}

The authors declare no conflict of interest.

\section{References}

1. Abramsky T, Devries KM, Michau L, et al. Ecological pathways to prevention: How does the SASA! community mobilisation model work to prevent physical intimate partner violence against women? BMC Public Health 2016; 16: 339.

2. Adjah ESO, Agbemafle I. Determinants of domestic violence against women in Ghana. BMC Public Health 2016; 16: 368.

3. Al-Natour A, Qandil A, Gillespie G. Nurses' roles in screening for intimate partner violence: a phenomenological study. Int Nurs Rev 2016; 63: 422-428.

4. Alvarez CP, Davidson PM, Fleming C, et al. Elements of effective interventions for addressing intimate partner violence in Latina women: A systematic review. PLoS One 2016; 11: e0160518.

5. Amini L, Daneshparvar H, Gharaee B, et al. The relationship between dimensions of domestic violence and social structural determinants of health in women. J Mazandaran Univ Med Sci 2014; 24: 131-134.

6. Beeble ML, Bybee D, Sullivan CM, et al. Main, mediating, and moderating effects of social support on the wellbeing of survivors of intimate partner violence across 2 years. J Consult Clin Psychol 2009; 77: 718-729.

7. Bott S, Morrison A, Ellsberg M. Preventing and responding to gender-based violence in middle and low-income countries: a global review and analysis [cited 2005 June]. Available from: https://openknowledge.worldbank.org/ handle/10986/8210.

8. Bradbury-Jones C, Clark MT, Parry J, et al. Development of a practice framework for improving nurses' responses to intimate partner violence. J Clin Nurs 2017; 26: 24952502.

9. Carvalho MR, Oliveira JF, Gomes NP, et al. Coping strategies for domestic violence: testimony of women involved with drugs [cited 2019 Apr 18]. Available from https://www.scielo.br/scielo.php?script=sci_arttext\&pi$d=$ S1414-81452019000200223.

10. Chepuka L, Taegtmeyer M, Chorwe-Sungani G, et al. Perceptions of the mental health impact of intimate partner 
violence and health service responses in Malawi. Glob Health Act 2014; 7: 24816.

11. Crombie N, Hooker L, Reisenhofer S. Nurse and midwifery education and intimate partner violence: a scoping review. J Clin Nurs 2017; 26: 2100-2125.

12. Dehghani-Firoozabadi ES, Mohtashami J, AtashzadehShoorideh $\mathrm{F}$, et al. Correlation between religious attitude and resiliency of women under domestic violence. Glob J Health Sci 2017; 9: 199-208.

13. Dicola D, Spaar E. Intimate partner violence. Am Fam Physician 2016; 94: 646-651.

14. Ferrari G, Feder G, Agnew-Davies R, et al. Psychological advocacy towards healing (PATH): a randomized controlled trial of a psychological intervention in a domestic violence service setting. PLoS One 2018; 13: e0205485.

15. García-Moreno C, Hegarty K, d'Oliveira AFL, et al. The health-systems response to violence against women. Lancet 2015; 385: 1567-1579.

16. Garrusi B, Nakhaee N, Zangiabadi M. Domestic violence: frequency and women's perception in Iran (IR). J Appl Sci 2008; 8: 340-345.

17. Golu F. Predictors of domestic violence-comparative analysis. Proc-Soc Behav Sci 2014; 127: 611-615.

18. Gómez-Fernández MA, Goberna-Tricas J, Payà-Sánchez M. Intimate partner violence as a subject of study during the training of nurses and midwives in Catalonia (Spain): a qualitative study. Nurs Educ Pract 2017; 27: 13-21.

19. Graneheim UH, Lundman B. Qualitative content analysis in nursing research: concepts, procedures and measures to achieve trustworthiness. Nurse Educ Today 2004; 24: 105-112.

20. Harvey S, Lees S, Mshana G, et al. A cluster randomized controlled trial to assess the impact on intimate partner violence of a 10-session participatory gender training curriculum delivered to women taking part in a groupbased microfinance loan scheme in Tanzania (MAISHA CRT01): study protocol. BMC Women Health 2018; 18: 55.

21. Itimi K, Dienye PO, Gbeneol PK. Intimate partner violence and associated coping strategies among women in a primary care clinic in Port Harcourt, Nigeria. J Fam Med Primary Care 2014; 3: 193-198.

22. Jack SM, Ford-Gilboe M, Wathen CN, et al. Development of a nurse home visitation intervention for intimate partner violence. BMC Health Serv Res 2012; 12: 50.

23. Kalra N, Di Tanna GL, García-Moreno C. Training healthcare providers to respond to intimate partner violence against women. Cochrane Database Syst Rev 2017; 2017.

24. Khangholi ZA, Mohtashami J, Hosseini S, et al. Investigation of rate of domestic violence against women presenting to legal medicine centers and its coping methods: a descriptive study. Crescent J Med Biol Sci 2019; 6: 164-169.

25. Khatri R, Pandey B. Causes of violence against women: a qualitative study at Bardiya district. Health Prospect 2013; 12: 10-14

26. Krishnan S, Subbiah K, Khanum S, et al. An intergenerational women's empowerment intervention to mitigate domestic violence: results of a pilot study in Bengaluru, India. Violence Against Women 2012; 18: 346-370.

27. Lincoln YS, Guba EG. But is it rigorous? Trustworthiness and authenticity in naturalistic evaluation. New Direct Program Eval 1986; 30: 73-84.

28. McCloskey LA, Lichter E, Williams C, et al. Assessing intimate partner violence in health care settings leads to women's receipt of interventions and improved health. Public Health Rep 2006; 121: 435-444.
29. McFarlane JM, Groff JY, O’Brien JA, et al. Secondary pre vention of intimate partner violence: a randomized controlled trial. Nurs Res 2006; 55: 52-61.

30. Noorisanchooli H, Asadibidmeshki E, Rahnama M, et al. The employed women's experience of spousal violence: a qualitative study. Med Sci 2018; 22: 560-565.

31. Pallitto C, García-Moreno C, Stöeckl H, et al. Testing a counselling intervention in antenatal care for women experiencing partner violence: a study protocol for a randomized controlled trial in Johannesburg, South Africa. BMC Health Serv Res 2016; 16: 630.

32. Polit DF, Beck CT. Nursing research: generating and assessing evidence for nursing practice. Lippincott Williams \& Wilkins, Philadelphia 2012.

33. Pouraboli B, Abedi HA, Abbaszadeh A, et al. Disease concealment: Experiences of thalassemia patients and their caregivers. Med-Surg Nurs J 2014; 3: 121-128.

34. Sadeghi-Fasaee S. Domestic violence and women's strategies in the face of it. J Soc Issue 2010; 1: 107-142.

35. Semahegn A, Torpey K, Manu A, et al. Community based intervention to prevent domestic violence against women in the reproductive age in Northwestern Ethiopia: a protocol for quasi-experimental study. Reproduct Health 2017; 14: 155.

36. Shuib R, Endut N, Ali SH, et al. Domestic violence and women's well-being in Malaysia: Issues and challenges conducting a national study using the WHO multi-country questionnaire on women's health and domestic violence against women. Proc Soc Behav Sci 2013; 91: 475488.

37. Sigalla GN, Rasch V, Gammeltoft T, et al. Social support and intimate partner violence during pregnancy among women attending antenatal care in Moshi Municipality, Northern Tanzania. BMC Public Health 2013; 17: 240.

38. Stover CS, Meadow AL, Kaufman J. Interventions for intimate partner violence: review and implications for evidence-based practice. Prof Psychol Res Pract 2009; 40: 223.

39. Taherkhani S, Negarandeh R, Simbar M, et al. Iranian women's strategies for coping with domestic violence. Nurs Midwifery Stud 2016; 5: e33124.

40. Tiwari A, Leung WC, Leung T, et al. A randomised controlled trial of empowerment training for Chinese abused pregnant women in Hong Kong. BJOG Int J Obst Gynaecol 2005; 112: 1249-1256.

41. Uzun GO, Uzunboylu $\mathrm{H}$. A survey regarding of domestic violence againts women. Proc-Soc Behav Sci 2015; 190: 24-31.

42. Vakily M, Noroozi M, Yamani N. Comparing the effect of group-based and compact disk-based training on midwives' knowledge and attitude toward domestic violence in women of reproductive age. J Educ Health Promot 2017; 6: 70.

43. WHO. Violence against women [cited 2014]. Available from: http://www.who.int/mediacentre/factsheets/fs239/en/. 